\title{
Pulmonary valve replacement: A new paradigm
}

\author{
Carl L. Backer, MD
}

From the Division of Cardiovascular-Thoracic Surgery, Ann \& Robert H. Lurie Children's Hospital of Chicago, Chicago, Ill; and Northwestern University Feinberg School of Medicine, Chicago, Ill.

Disclosures: C.L.B. is a consultant to W.L. Gore \& Associates, Flagstaff, Arizona.

Received for publication Dec 11, 2017; accepted for publication Dec 21, 2017; available ahead of print Feb 1, 2018.

Address for reprints: Carl L. Backer, MD, Cardiovascular-Thoracic Surgery, Ann \& Robert H. Lurie Children's Hospital of Chicago, 225 E. Chicago Ave, mc 22, Chicago, IL 60611 (E-mail: cbacker@luriechildrens.org). J Thorac Cardiovasc Surg 2018;155:1710-1

0022-5223/\$36.00

Copyright (c) 2018 by The American Association for Thoracic Surgery

https://doi.org/10.1016/j.jtcvs.2017.12.092

Pulmonary valve insufficiency after repair of tetralogy of Fallot is a common occurrence and can lead to right ventricular dysfunction, tricuspid valve insufficiency, atrial and ventricular arrhythmias, and eventual right heart failure. Pulmonary valve replacement has been shown to mitigate these undesirable sequelae, but eventually pulmonary valves fail and require (before now) surgical replacement. The decision as to when to recommend implantation of a pulmonary valve has been based on the competing risks of intervening before it is too late, but recognizing that once a valve has been inserted it will require eventual replacement.

The multicenter study reported by Cabalka and colleagues $^{1}$ brings us into a new paradigm for these patients. They report 100 patients who underwent transcatheter pulmonary valve insertion using as a "landing zone" the previous surgically placed bioprosthetic pulmonary valve. There were no major periprocedural complications. Mean right ventricular outflow tract gradient was reduced from 29 to $16 \mathrm{~mm} \mathrm{Hg}$. Pulmonary valve competency was restored in all but 1 patient. Although the follow-up is short (median, 1 year) no patients have required reintervention on the transcatheter pulmonary valve. Although there are anecdotal reports of endocarditis after transcatheter valve insertion, only 1 patient in this series developed endocarditis, and that was successfully managed with medical therapy.

The ability of pediatric interventional cardiologists to successfully place transcatheter valves with a minimum of complications has changed the paradigm for the care of patients with pulmonary insufficiency after repair of tetralogy of Fallot. Although we do not currently know the eventual longevity of these transcatheter valves, certainly every additional year of a functioning transcatheter valve is an important additional year. This addition to our armamentarium will lead to an earlier recommendation for surgical placement of the first valve knowing that the second valve and perhaps the third valve can be placed in a transcatheter fashion.

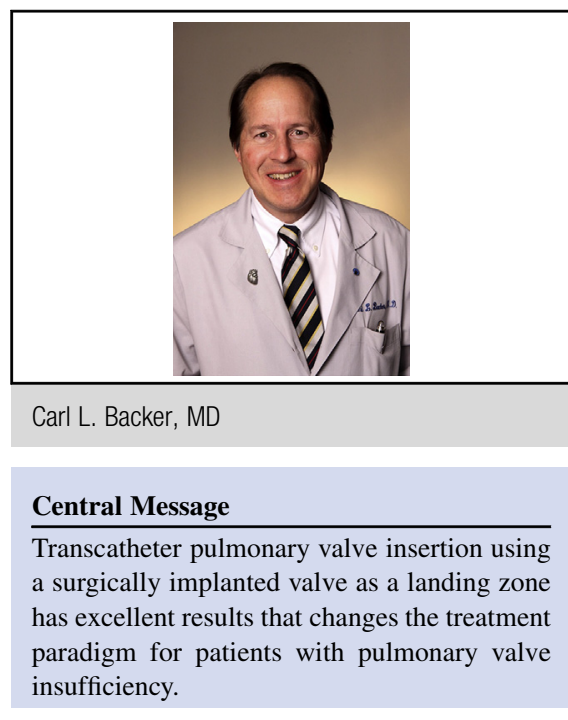

See Article page 1712 .

The excellent results with this 2-step strategy must be carefully considered when contemplating the placement of transcatheter valves in a native tetralogy outflow tract without the "landing zone" of a surgically placed bioprosthetic pulmonary valve. A total of 100 consecutive cases in this series from 10 separate centers with outstanding results seems to demonstrate that the "2-step" strategy is one that will be difficult to compete against. Certainly in my experience and in anecdotal reports from other surgeons, transcatheter valve insertion without a "landing zone" is a recipe for complications requiring urgent surgical intervention. The other competing strategy for these patients is mechanical pulmonary valve insertion. Although these valves last longer, they require anticoagulation and the patients are not candidates for later transcatheter valve insertion.

Collaboration between cardiac surgeons and cardiologists to optimize the lifetime management of patients with tetralogy of Fallot with pulmonary insufficiency now has a new paradigm. This new strategy will optimize right ventricular function, decrease arrhythmias, and lead to less right heart failure in this patient population. Surgeons need to implant valves that optimize the potential for late successful placement of a transcatheter valve. Careful follow-up of these patients will ultimately lead to new guidelines for the timing of pulmonary valve insertion. 


\section{Reference}

1. Cabalka AK, Asnes JD, Balzer DT, Cheatham JP, Gillespie MJ, Jones TK, et al. Transcatheter pulmonary valve replacement using the Melody valve for treatment of dysfunctional surgical bioprostheses: a multicenter study. J Thorac Cardiovasc Surg. 2018;155:1712-24. 